University of Massachusetts Amherst

ScholarWorks@UMass Amherst

Chemistry Department Faculty Publication Series

Chemistry

1984

\title{
Potential Use of a Terbium-Transferrin Complex as a Label in an Immunoassay for Gentamicin
}

\author{
N.J. Wilmott \\ Loughborough University of Technology \\ J. N. Miller \\ Loughborough University of Technology \\ Julian Tyson \\ University of Massachusetts Amherst
}

Follow this and additional works at: https://scholarworks.umass.edu/chem_faculty_pubs

Part of the Chemistry Commons

\section{Recommended Citation}

Wilmott, N. J.; Miller, J. N.; and Tyson, Julian, "Potential Use of a Terbium-Transferrin Complex as a Label in an Immunoassay for Gentamicin" (1984). Analyst. 1287.

Retrieved from https://scholarworks.umass.edu/chem_faculty_pubs/1287 


\title{
Potential Use of a Terbium - Transferrin Complex as a Label in an Immunoassay for Gentamicin
}

\author{
Nichola J. Wilmott, James N. Miller and Julian F. Tyson \\ Chemistry Department, Loughborough University of Technology, Loughborough, Leicestershire, \\ LE11 3TU, UK
}

\begin{abstract}
A study has been made of the potential use of a terbium - transferrin complex as a non-isotopic label in the immunoassay determination of the antibiotic gentamicin. The fluorescence properties of the complex have been characterised. The labelled gentamicin was formed using a controlled carbodiimide reaction, conditions being chosen to produce a gentamicin-bound complex containing the correct amount of gentamicin for use in a competitive binding assay. Recognition of the gentamicin-bound complex by antisera to gentamicin was verified using a standard radioimmunoassay for gentamicin.
\end{abstract}

Keywords: Fluorescence immunoassay; gentamicin assay; terbium - transferrin label

The last decade has seen several attempts to develop non-isotopic immunoassays with a view to replacing radioimmunoassay methods which have many well documented disadvantages.

Several approaches have been made to the use of metal ions as labels in immunoassay: Cais ${ }^{1,2}$ used derivatives of sandwich compounds such as ferrocene to label steroids and other molecules, detection of the labelled species being carried out by atomic-absorption spectrometry. These labels were relatively insensitive in immunoassays, no doubt partly because the metals used ( $\mathrm{Fe}, \mathrm{Mn}$, etc.) occur at high concentrations in biological samples. Cais subsequently synthesised metallohaptens by the mercuration of steroid estrogens, ${ }^{3}$ where the metal atoms were introduced directly into the molecule of the antigen, and by the formation of cymatrene complexes containing manganese.$^{4}$ Leuvering et al.${ }^{5}$ have studied the use of colloidal coating with atomic-absorption spectrometric determination.

In this paper we report the potential use of lanthanide ions as labels in immunoassay. In principle, lanthanide ion complexes should form useful labels in immunoassay as they are highly fluorescent and the concentration of lanthanide ions in biological samples is extremely low. In particular, a study has been made of the potential of the complex between terbium and transferrin as a label in the immunoassay of gentamicin.

Human serum transferrin has the function in the body of binding and transporting iron in the plasma in the form of $\mathrm{Fe}^{3+}$. Transferrin binds two iron(III) ions per molecule in specific sites on the polypeptide chain, but in the absence of iron these sites may be occupied by lanthanide ions to form highly fluorescent complexes.

Gentamicin is an aminoglycosidic antibiotic used in the treatment of severe Gram-negative bacteria. Its optimum therapeutic concentration in human serum is $4-12 \mathrm{mg}^{-1}$ (9-20 $\left.\mu \mathrm{mol} \mathrm{1}^{-1}\right)$, maintained by intramuscular injection: $0.8 \mathrm{mg}$ per kilogram body mass every $8 \mathrm{~h}$. It is extremely effective but has a low therapeutic ratio and therefore its level must be monitored by a simple, rapid and reliable method.

\section{Experimental}

\section{Apparatus}

All fluorescence spectra were measured on a Perkin-Elmer Model LS-5 luminescence spectrometer fitted with an R928 photomultiplier. Data were recorded using a Perkin-Elmer Model 3600 data station using PECLS II applications software, which permitted the calculation of corrected spectra and phosphorescence lifetimes.

\section{Reagents}

Terbium chloride and glycine were obtained from Aldrich Chemical Co, and iron-free transferrin, gentamicin sulphate and 1-ethyl-3-(3-dimethylaminopropyl)carbodiimide hydrochloride from Sigma London Chemical Co.

All radioimmunoassays were carried out using a radioimmunoassay kit supplied by RIA (UK) Ltd.

Antibodies used in the antibody binding experiments were supplied by Sigma Chemical Co. Ltd.

\section{Fluorescence of Terbium and the Terbium - Transferrin Complex}

Fluorescence spectra of terbium chloride in aqueous solution and a terbium - transferrin complex in Tris - hydrochloric acid hydrogen carbonate buffer ${ }^{6}$ were recorded.

The limits of detection of the complex and its fluorescence lifetime (the time for the fluorescence of a sample to decay to the background level) were also determined.

The complex for the above experiments was prepared by the addition of aqueous terbium chloride to iron-free transferrin in Tris - hydrochloric acid - hydrogen carbonate buffer in the molar ratio $2: 1$.

\section{Linkage of Gentamicin to Transferrin}

The linkage of gentamicin to transferrin was achieved using a carbodiimide reagent. In this procedure a free acid group on the transferrin molecule is linked to a free amino group on the gentamicin. Experiments were carried out using 1-ethyl-3-(3dimethylaminopropyl)carbodiimide hydrochloride in aqueous solution.

Iron-free transferrin $(30 \mathrm{mg})$, gentamicin $(60 \mathrm{mg})$ and the carbodiimide $(400 \mathrm{mg})$ were incubated at room temperature in distilled water $(2.5 \mathrm{ml})$ for varying lengths of time before separation of the protein fraction on a Sephadex G-25 column by elution with Tris - hydrochloric acid - hydrogen carbonate buffer ( $\mathrm{pH} \mathrm{8.5).} \mathrm{Verification} \mathrm{of} \mathrm{gentamicin} \mathrm{binding} \mathrm{was}$ carried out using ${ }^{125}$ I-labelled gentamicin.

The carbodiimide procedure was repeated using various glycine to gentamicin ratios for the gentamicin in the original experiment, the reaction time being $5 \mathrm{~min}$ in each instance. The reaction products were subsequently analysed for their gentamicin content by radioimmunoassay and their fluorescence was measured after terbium binding. 


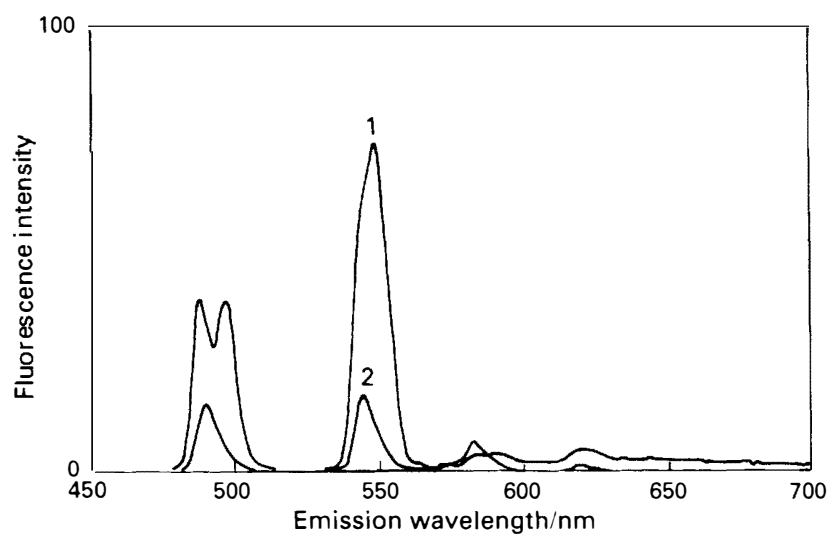

Fig. 1. Emission spectra of terbium chloride and a terbium transferrin complex. $1,10^{-5} \mathrm{M}$ terbium - transferrin complex; and 2 $100 \mathrm{~mm}$ terbium chloride

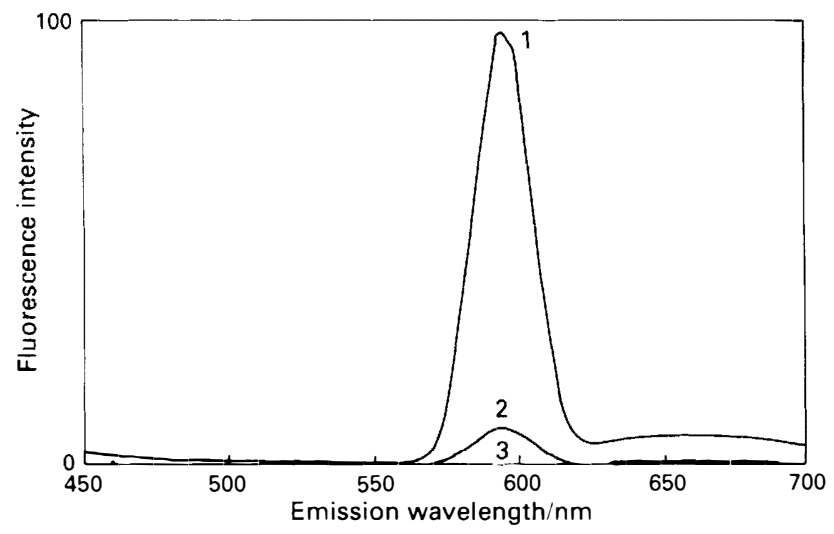

Fig. 2. Emission spectra of $1: 100$ human serum with various delay times: $1,0.01 ; 2,0.02$; and $3,0.05 \mathrm{~ms}$

\section{Titration of the Gentamicin - Glycine Bound Complex against Anti-gentamicin}

The effect on the fluorescence of the complex in various concentrations of antiserum to gentamicin (raised in rabbit) was investigated using the double antibody technique with anti-rabbit $\mathrm{IgG}$. Fluorescence measurements were taken following incubation, precipitation and centrifugation.

\section{Results and Discussion}

\section{Fluorescence of Terbium and the Terbium - Transferrin Complex}

Fig. 1 shows an enhancement of the fluorescence intensity of terbium of the order of $10^{5}$ when the complex is excited at 295 $\mathrm{nm}$. This fluorescence enhancement is due to an intramolecular energy transfer process within the complex. This is demonstrated by the fact that the complex excitation is in the region of the ligand absorbing region whereas the emission is typical of the "line" emission of the terbium ion.

The limit of detection of the complex was shown to be $10^{-7}$ $\mathrm{M}$ with respect to transferrin, a result which suggests that the complex has a suitable fluorescence intensity for use as a label in the analysis of drugs by immunoassay.

The complex had a lifetime of $1.25 \mathrm{~ms}$. Subsequent delayed fluorescence experiments (Fig. 2) showed that interference due to scattered light from blood serum could be reduced to zero with a delay gate of $0.05 \mathrm{~ms}$. The subsequent decrease in fluorescence intensity of the complex was less than $5 \%$. The following instrumental conditions were thus chosen: excitation slit, $5.0 \mathrm{~nm}$; emission slit, $5.0 \mathrm{~nm}$; excitation wavelength $295 \mathrm{~nm}$; delay time, $0.05 \mathrm{~ms}$; gate time, $5.0 \mathrm{~ms}$; and fixed-scale mode.
Table 1. Results of analysis of complex for gentamicin by radioimmunoassay

\begin{tabular}{|c|c|c|c|}
\hline Analyte & & C.p.s. & $\begin{array}{c}\text { Gentamicin } \\
\text { determined/ } \\
\mu \mathrm{g} \mathrm{ml}^{-1}\end{array}$ \\
\hline $0 \mu \mathrm{g} \mathrm{ml}^{-1}$ standard & . & 42039 & \\
\hline $1 \mu \mathrm{g} \mathrm{ml}^{-1}$ standard & . & 36557 & \\
\hline $2 \mu \mathrm{g} \mathrm{ml}^{-1}$ standard & $\cdots$ & 28737 & \\
\hline $4 \mu \mathrm{g} \mathrm{ml}^{-1}$ standard & . & 16771 & \\
\hline $8 \mu \mathrm{g} \mathrm{ml}^{-1}$ standard & & 9860 & \\
\hline $16 \mu \mathrm{g} \mathrm{ml}^{-1}$ standard $\ldots$ & . & 6481 & \\
\hline \multicolumn{4}{|l|}{ Glycine : gentamicin ratio- } \\
\hline $0: 20$ & & 1023 & \\
\hline $2: 18$ & & 540 & \\
\hline $5: 15$ & & 1073 & $>16$ \\
\hline $10: 10$ & & 1870 & \\
\hline $15: 5$ & & 7497 & $8-16$ \\
\hline $18: 2$ & & 11493 & $4-8$ \\
\hline
\end{tabular}

Table 2. Results of stability tests on the glycine - gentamicin bound complex

\begin{tabular}{ccc} 
Time after & $\begin{array}{c}\text { Fluorescence } \\
\text { intensity in } \\
\text { buffer }\end{array}$ & $\begin{array}{c}\text { Fluorescence } \\
\text { intensity in } \\
1: 100 \text { serum }\end{array}$ \\
$0 \mathrm{~min}$ & 104.95 & 64.19 \\
$5 \mathrm{~min}$ & 109.55 & 67.48 \\
$10 \mathrm{~min}$ & 103.50 & 71.19 \\
$15 \mathrm{~min}$ & 104.25 & 72.84 \\
$30 \mathrm{~min}$ & 107.10 & 74.07 \\
$45 \mathrm{~min}$ & 106.20 & 74.80 \\
$1 \mathrm{~h}$ & 107.05 & 77.45 \\
$24 \mathrm{~h}$ & 109.00 & 77.46 \\
$7 \mathrm{~d}$ & 110.95 & 77.46 \\
\hline
\end{tabular}

\section{Linkage of Gentamicin to Transferrin}

The experiments carried out using $125 \mathrm{I}$-labelled gentamicin showed that with reaction times from $5 \mathrm{~min}$ to $1 \mathrm{~h}$ substantial gentamicin binding was occurring.

\section{Analysis of the Gentamicin Content of Complexes with Substituted Glycine}

Table 1 shows the amount of gentamicin bound per millilitre of solution for various reaction mixtures as analysed by standard radioimmunoassay for gentamicin.

It can be seen that gentamicin - glycine bound complexes with glycine to gentamicin ratios of less than $15: 5$ have too high a gentamicin content for use in a competitive binding assay for the therapeutic blood concentration range of gentamicin $\left(4-12 \mu \mathrm{g} \mathrm{ml}^{-1}\right)$.

Fluorescence measurements on the complexes modified by glycine and gentamicin showed a decrease in fluorescence intensity of less than $5 \%$ compared with the original terbium transferrin complex.

\section{Stability of the Gentamicin - Glycine Bound Complex}

It can be seen from Table 2 that a complex containing a glycine to gentamicin ratio of $9: 1$ is stable in buffer and buffer containing $1: 100$ human serum over a period of at least $7 \mathrm{~d}$.

Absorbance measurements at $295 \mathrm{~nm}$ showed that the decrease in fluorescence intensity in $1: 100$ serum was due to the increased absorbance of the solution at that wavelength.

\section{Titration of the Glycine - Gentamicin Complex against Anti-gentamicin}

Titration of the complex against anti-gentamicin (Fig. 3) shows good antibody recognition of the complex, the curve shape being typical of such a dilution analysis. 


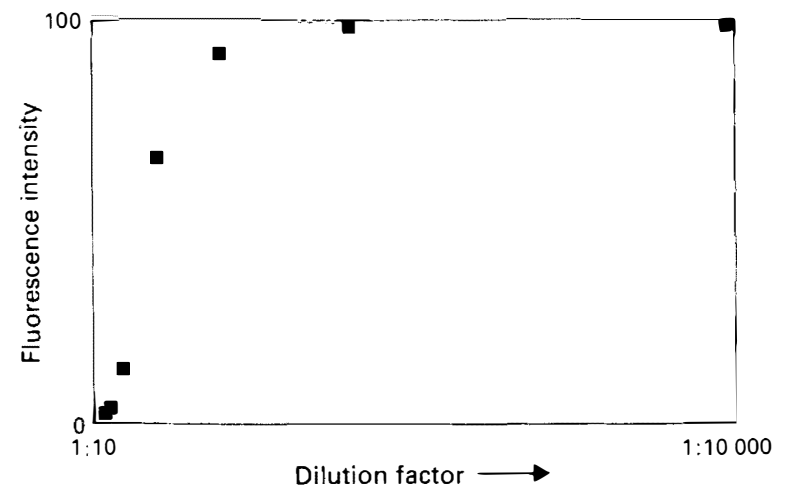

Fig. 3. Antibody dilution analysis of the terbium - transferrin gentamicin - glycine complex

\section{Conclusion}

The results suggest that a glycine - gentamicin bound terbium transferrin complex has potential as a non-isotopic immunoassay label. However, it is felt that careful consideration should be given to the final format of the immunoassay, owing to possible effects of the macromolecular label. At present the most suitable separation methods are being established.

Although the terbium - transferrin complex shows potential as an alternative label in immunoassay, it has the disadvantage of sensitivity suitable only for the detection of drugs and not hormones. One means of increasing the sensitivity is to increase the fluorescence intensity. Further study of the intramolecular energy transfer process ${ }^{7,8}$ such as are involved in the terbium - transferrin complex might prove useful in this respect. The bifunctional reagents that would be suitable are of the type described by Meares and co-workers, $, 9,10$ which have "EDTA" groups and groups suitable for binding to amino acids at opposite extremities of the molecule. Another alternative is the use of bifunctional reagents where the chelating agent is "iminodiacetic acid." 11,12

The authors thank the Trustees of the Analytical Chemistry Trust Fund of the Royal Society of Chemistry for the award of an SAC Research Studentship.

\section{References}

1. Cais, M., Nature (London), 1977, 270, 534.

2. Cais, M., US Pat., $4205952,1980$.

3. Cais, M., Actual. Chim., 1979, No. 7,14.

4. Cais, M., Bull. Soc. Chim. Belg., 1981, 90, 27.

5. Leuvering, J. H. W., Thal, P. J. H. M., Van der Waart, M., and Schuurs, A. H. W. M., J. Immunoassay, 1980, 1, 77.

6. Luk, C. K., Biochemistry, 1971, 10, 2838.

7. Weissman, S. I., J. Chem. Phys., 1942, 10, 214.

8. Crosby, G. A., Whan, R. E., and Aline, R. M., J. Chem. Phys., 1961, 34, 743.

9. Sundberg, M. W., Meares, C. F., Goodwin, D. A., and Diamanti, C. A., J. Med. Chem., 1974, 17, 1304.

10. Yeh, S. M., Sherman, D. G., and Meares, C. F., Anal. Biochem., 1979, 100, 152.

11. De Maulipied, A., J. Chem. Soc., 1905, 87, 439.

12. Johnson, C., J. Am. Chem. Soc., 1911, 33, 749. 